\title{
Considérations sur les tâches des Sociétés nationales de la Croix-Rouge dans le domaine de la sauvegarde des populations et du secours aux gazés de guerre
}

\author{
Zangger, Heinrich
}

\begin{abstract}
A l'occasion de la XVe Conférence de la Croix-Rouge, le Comité international a adressé à chacune des Sociétés nationales de la Croix-Rouge une lettre par laquelle il lui exprimait le désir de recevoir un rapport, aussi détaillé que possible, sur son activité dans le domaine de la protection des populations civiles contre les effets de la guerre aérienne, chimique, incendiaire, etc
\end{abstract}

DOI: https://doi.org/10.1017/s1026881200184934

Posted at the Zurich Open Repository and Archive, University of Zurich ZORA URL: https://doi.org/10.5167/uzh-154021

Journal Article

Published Version

Originally published at:

Zangger, Heinrich (1934). Considérations sur les tâches des Sociétés nationales de la Croix-Rouge dans le domaine de la sauvegarde des populations et du secours aux gazés de guerre. International Review of the Red Cross, 16(190):789-805.

DOI: https://doi.org/10.1017/s1026881200184934 


\section{REVUE INTERNATIONALE de la Croix-Rouge}

\section{Heinrich ZANGGER,}

professeur à l'Université et directeur de l'Institut de médecine légale de Zurich,

membre du Comité international de la Croix-Rouge.

Considérations sur les tâches des Sociétés nationales de la Croix-Rouge dans le domaine de la sauvegarde des populations et du secours aux gazés de guerre.

I.

A l'occasion de la XVe Conférence de la Croix-Rouge, le Comité international a adressé à chacune des Sociétés nationales de la Croix-Rouge une lettre pár laquelle il lui exprimait le désir de recevoir un rapport, aussi détaillé que possible, sur son activité dans le domaine de la protection des populations civiles contre les effets de la guerre aérienne, chimique, incendiaire, etc.

Cette lettre, qui porte la date du 10 janvier 1934, a été publiée dans la Revue internationale ${ }^{1}$.

Vingt-sept Sociétés nationales ont bien voulu répondre au vou du Comité international; les rapports qu'elles ont envoyés ont été publiés dans la Revue internationale ${ }^{2}$ et ils sont reproduits dans le document no 14 que le

1 Janvier 1934, pp. 49-50.

2 Mai 1934, pp. 388-405; juin pp. 491-506; juillet pp. 587-612; août pp. 663-668. 


\section{Heinrich Zangger.}

Comité international présentera à Tokio, en octobre prochain, sous le titre :

"Protection des populations civiles contre la guerre aérochimique ".

L'examen attentif des rapports des Sociétés nationales permet de dégager les faits suivants :

1) De nombreuses Sociétés nationales organisent ladite protection suivant les besoins des diverses régions de leur pays.

Aussi les organisations et la législation diffèrent-elles d'un pays à l'autre. Mais on peut remarquer que l'œuvre de sauvegarde tend de plus en plus à se rattacher aux Ministères de la guerre et de l'intérieur.

2) La protection du territoire et des populations civiles contre les effets de la guerre chimique, que nous envisageons plus spécialement ici, étant partout prévue ou en voie d'organisation, et devant être assurée avec célérité, une attaque par surprise, avec gaz de guerre, ne serait, au début d'un conflit armé, ni aussi foudroyante ni aussi efficace que sans cette protection.

Mais une question inquiétante se pose: Dans quelle mesure une défense systématique étendue rend-elle moins redoutable la surprise par laquelle un agresseur, recourant en même temps à tous les moyens de guerre, tenterait un coup décisif en semant la panique dans les grandes villes?

3) Il semble, en principe, que les défenses préparées, soit par l'organisation civile locale, soit par l'organisation militaire, sont partout presque les mêmes et que dès le début d'un conflit armé, les moyens de défense passive (guet, alarme, etc.) passeraient automatiquement aux mains des militaires.

4) A la suite des déceptions subies en matière de désarmement, on reste universellement convaincu qu'on doit préparer la défense contre les attaques de gaz, et 


\section{Sauvegarde des populations et secours aux gazés de guerre.}

tout le monde tient les gaz de guerre pour la plus puissante, la plus destructive et la plus démoralisante de toutes les armes.

En menaçant, dès le premier instant, les grandes villes et les centres industriels, cette arme paraît, plus que toute autre évolution technique de la guerre, devoir modifier les tâches de la Croix-Rouge et les modes de sa collaboration.

Il faut prévoir : la préparation de la défense passive des populations civiles des villes et des territoires proches des frontières, le danger des gaz restant potentiel pour tout le territoire du pays ; l'organisation du sauvetage et du transport des gazés dans les villes, hors de la zone d'action des gaz toxiques, des incendies; la collaboration des organisations municipales de sauvetage, des sapeurspompiers, des corps semi-militaires et des organisations de Croix-Rouge : premiers soins au front, transport des blessés et des gazés, etc.

Pour réaliser, au front, ses tâches traditionnelles: relever, soigner, transporter les blessés, la Croix-Rouge se trouve désormais dans des conditions nouvelles en raison du danger des gaz qui est partout présent et modifie tout :

Elle doit, notamment, sauver les personnes intoxiquées par les gaz et leur donner les premiers soins alors que l'atmosphère reste malsaine, puis s'efforcer de détruire les toxiques persistants comme l'ypérite.

Ces tâches ne peuvent s'accomplir dans toute la zone où les gaz restent dangereusement concentrés qu'avec un matériel de protection individuel mobile, peu pesant, interchangeable entre le sauveteur et l'intoxiqué. Ces moyens de protection individuels connus sont le masque filtrant et le masque isolant à oxygène. Grâce à cet appareil l'isolation est complète et l'individu peut respirer même dans les milieux chargés de gaz toxiques ou de combinaisons chimiques que des masques filtrants laisseraient en 


\section{Heinrich Zangger.}

partie passer. En outre, on ne peut pas recourir à la protection de choix que l'industrie procure contre les gaz toxiques, telles que les installations plus ou moins permanentes de renouvellement d'air puisqu'on ne dispose pas de sources d'air non contaminé. (Une situation comparable : il faut aussi protéger individuellement les sauveteurs, quand ils vont au secours d'électrocutés, dont les corps sont encore traversés par des courants électriques, afin de les prémunir eux-mêmes contre le danger de ces courants.)

Les réponses des Sociétés nationales ne traitent pas par le détail le point spécial de la protection individuelle, car depuis la guerre, les gaz nouveaux comme la perfection des filtres protecteurs sont envisagés comme des secrets absolus et des états-majors et des industries de guerre attachées aux Etats et des grandes fabriques de masques.

Personne ne peut savoir dans quelle mesure les inventions nouvelles sont connues des pays étrangers ou de leurs industries.

II.

L'extension de la guerre chimique aura pour effet de modifier les lois qui règlent l'activité des différentes organisations de la Croix-Rouge.

Les réponses des Sociétés nationales nous imposent le devoir d'examiner dans quelle mesure elles peuvent adapter leur action aux circonstances, d'après les expériences qui ont été faites pendant la guerre, ou avec les gaz toxiques industriels, ou après les catastrophes dues aux gaz nocifs de l'industrie. On doit se demander aussi sur quels points de leur activité il leur sera possible d'échanger conseils et expériences avec les Sociétés similaires de la Croix-Rouge.

Les conseils basés sur les connaissances acquises à un moment donné - surtout en ce qui concerne les masques à filtre - ne peuvent que peu de temps se rapporter aux dangers réels; en revanche les principes 


\section{Sauvegarde des populations \\ et secours aux gazés de guerre.}

généraux de protection, tel celui qui règle le traitement des gazés, resteront très probablement à peu près constants. De plus, quiconque se permet aujourd'hui de publier sur le sujet des masques doit se rendre compte des limites de ses connaissances et de la responsabilité qu'il assume, soit à cause de sa position comme savant, soit à cause du prestige de l'organisation qu'il représente. Il doit, en outre, s'aviser qu'il ne peut pas divulguer toutes les connaissances qu'il possède. En voici les raisons essentielles: Depuis 20 ans, des faits scientifiques physicochimiques se sont accumulés, qui servent de base à l'efficacité des filtres: par exemple, les actions de surfaces. C'est la superposition de grandes séries de fonctions qui réalise les fonctions spécifiques cherchées. Or, ces combinaisons et superpositions de fonctions en séries n'appartiennent pas aujourd'hui au domaine public. Elles résultent des expériences effectuées par l'industrie des filtres, ainsi que de nombreuses expériences faites par l'industrie en général, et les ministères de la guerre détiennent une masse de secrets. Il n'existe probablement pas d'hommes disposant de connaissances étendues dans ce domaine qui ne soient pas tenus au secret absolu en qualité de militaires ou de fonctionnaires d'Etat, ou d'industriels. Pour la plupart des points capitaux en cette matière, le conflit moral des responsabilités se présente comme il ne l'a jamais été. Voici des personnes qui ont fait des expériences désastreuses en cherchant à secourir des intoxiqués, victimes d'une catastrophe ou d'une explosion; elles ont constaté l'insuffisance des moyens dont elles disposaient. Eh bien, devant elles se dresse, tel un moloch impassible, l'impérieuse obligation du secret.

Il faut en outre savoir que, dans les grandes industries, de grandes séries de connaissances très concrètes et techniquement formulées sont confiées aux archives secrètes des fabriques. 


\section{Heinrich Zangger.}

\section{III.}

Au sujet des masques anti-gaz, il y a des considérations générales qui doivent être soulignées.

a) On tend partout, activement, à organiser la protection individuelle comme la protection collective. Aussi les Sociétés nationales de la Croix-Rouge doiventelles se rappeler que la plupart de ceux qui désirent se procurer des moyens de protection sont exposés à des malentendus ou menacés par les suggestions de fabricants ou de commerçants peu consciencieux.

D'où cette première question : les Sociétés nationales peuvent-elles exercer une influence sur le contrôle et sur la vente des masques filtrants et de tous les moyens de protection préconisés par la réclame et par lesquels on voudrait se prémunir contre le péril des gaz de guerre ?

b) Des centaines d'entreprises ont été créées pour fabriquer des masques à gaz et pour les vendre, et de nombreux Etats n'exercent aucun contrôle à cet égard. Dans la crise qui sévit, beaucoup de gens sont très attirés par les nouvelles industries et les commerces nouveaux; certains même sont tentés d'exploiter l'angoisse des populations.

Les réponses des Sociétés nationales permettent de penser que beaucoup d'Etats établissent une législation en cette matière; dans d'autres pays, la production et même la vente des gaz de guerre, des masques et des filtres anti-gaz, bénéficie complètement de la liberté du commerce et de la production industrielle.

Dans l'intérêt de la population civile, il convient d'affirmer ici que les moyens destinés à la protection individuelle sont limités quant à leur action et quant à leur durée de conservation et que leur efficacité diminue avec le temps. L'acheteur, lui, ignore tout cela et il demeure incapable d'apprécier l'état de conservation d'un masque à gaz, car il dispose de moins de cri- 


\section{Sauvegarde des populations \\ et secours aux gazés de guerre.}

tères encore que pour les spécialités médicales. Les spécialités pharmaceutiques, en effet, sont en général contrôlées, leurs combinaisons chimiques sont enregistrées officiellement, voire indiquées sur l'enveloppe. Or, ce n'est que dans fort peu de grands Etats qu'on trouve dans le commerce des filtres anti-gaz officiellement contrôlés. Les seules garanties d'efficacité sont donc fournies par l'industrie qui produit ces moyens de protection individuelle.

Et ces considérations demeurent des sujets d'inquiétudes.

Comment garantir à la population civile et aux organisations de Croix-Rouge qu'elles peuvent en tout temps se procurer les moyens de protection qui seront les plus actifs et qui n'auront subi ni détérioration, ni usure, ni déformation ?

Comment, d'autre part, leur garantir que le commerce, plus ou moins favorisé du contrôle officiel, ne fera pas de profits non autorisés et déraisonnables?

Ni le personnel des Sociétés de la Croix-Rouge, ni la population civile ne sont à même de contrôler les divers appareils anti-gaz mis dans le commerce, appareils dont les qualités fort différentes échappent à l'appréciation, dont la durée est mal déterminée et dont le rôle, toujours limité, est mal connu.

S'ils ne le peuvent pas, c'est surtout parce que ces moyens de protection ne sont pas, comme les remèdes et les aliments, achetés en vue du lendemain, mais pour un temps indéterminé, imprévisible, peut-être lointain et pour des circonstances où le danger chimique sera devenu tout autre qu'on ne l'imagine aujourd'hui.

Ces raisons très diverses font comprendre que les moyens de protection individuelle lancés sans contrôle dans le commerce ne tiendront pas leurs promesses et n'assureront pas le secours qu'on aurait pu en attendre, si l'on avait veillé scientifiquement à leur conservation. Et 


\section{Heinrich Zangger.}

quoi de plus dangereux que d'accorder sa confiance à un moyen de protection individuelle qui ne présente pas de garantie?

Il faut avouer que cela explique les pertes de vies humaines inattendues qu'on a constatées dans les catastrophes industrielles et les explosions de gaz toxiques de moindre importance.

IV.

En principe, nous pouvons donner certains conseils et faire certaines prévisions: les masques anti-gaz mis en vente doivent être garantis pour des toxiques déterminés, pour des combinaisons et des concentrations de toxiques données, ainsi que pour le temps de leur emploi et la durée de leur fonctionnement.

Mais qui peut fournir ces garanties?

Si les Etats prévoient le contrôle des denrées alimentaires et des divers objets usuels, voire des parasiticides, il est bien évident que les moyens de protection basés sur la chimie doivent être contrôlés encore plus rigoureusement.

Dans certains Etats, de nouvelles Sociétés ou des filiales créées par des étrangers bénéficient de la liberté du commerce. Le danger des gaz redouté des familles fournit ainsi au commerce des moyens d'exploitation.

$$
\text { V. }
$$

Le danger de la guerre des gaz a conduit ou conduira sans doute les Sociétés nationales à se placer sous la protection des ministères de la guerre. Elles y trouveront des appuis pour prendre les mesures qui protègeront, au moins temporairement, leur personnel exposé au danger des gaz.

La partie purement mécanique d'un masque à gaz filtrant, peut être fabriquée et stockée si l'on est assuré qu'au moment d'une déclaration de guerre des filtres 


\section{Sauvegarde des populations \\ et secours aux gazés de guerre.}

efficaces appropriés seront mis à la disposition des organisations de secours.

Les autorités militaires compétentes pourront évidemment confier cette fabrication aux industriels compétents. Quant aux appareils à oxygène, ils pourront être également fabriqués et stockés, mais il faudra les contrôler périodiquement. En effet, les bombes d'oxygène sous pression qui, pendant des années, ne sont pas utilisées, se vident; au moment critique, elles n'ont plus d'oxygène. Que de fois n'en a-t-on pas fait l'expérience dans des situations moins graves et jusqu'à un certain point prévisibles (explosion de gaz, production de gaz délétères dans les fabriques, les mines, les tunnels!)

VI.

Dans chaque Etat on demeure convaincu que l'on possède des toxiques et des combinaisons de toxiques qui l'emporteront sur les moyens de défense et de protection de l'ennemi; types et combinaisons de gaz qui passent par les filtres et qui les détériorent. Et cette même conviction se retrouve dans tous les Etats. En outre, dans bien des pays, on a cherché, chacun le sait, des substances agressives vésicantes nouvelles. Or, au moment d'une attaque, une grande partie de la population civile se trouve en dehors des maisons; aussi est-il à craindre qu'elle ne soit pas protégée contre les effets de ces substances.

De tels faits conduisent alors à formuler le postulat suivant:

Une agression par surprise avec des toxiques connus, avec des toxiques inconnus et avec des mélanges de toxiques imprévus sera un moyen auquel on recourra toujours.

VII.

Depuis une dizaine d'années, des recherches systématiques et des découvertes fortuites ont donné de nouvelles 


\section{Heinrich Zangger.}

bases scientifiques au développement de la technique de l'agression avec des gaz de guerre. En plus des vésicants il est des substances nouvelles qui ne sont pas retenues par les filtres, et d'autres qui diminuent leur efficacité.

On a partout à peu près autant de chances de trouver des substances analogues.

Si le hasard m'a fait trouver, ces dernières années, et réaliser des types chimiques très différents, je manquerais du sens des réalités en n'admettant pas que dans les grands laboratoires de gaz de guerre et dans l'industrie des masques anti-gaz on a des chances bien plus nombreuses de faire de semblables découvertes. On serait inexcusable de ne pas tirer les conséquences de ces faits pour les organisations de Croix-Rouge.

Ces faits secrets lieront les Sociétés de la Croix-Rouge aux organisations militaires nationales puisque la protection individuelle doit prévoir les modifications continuelles de la technique militaire. D'autre part, ces faits - si l'on en juge bien, contribuent à mettre en évidence l'importance de la protection de la population par d'autres moyens de défense passive.

Voici comment en principe on peut assurer la protection par des moyens non-chimiques, et comment la Croix-Rouge peut agir en ce domaine:

a) Evacuation des grandes villes (femmes, enfants, malades). Difficultés dues aux saisons, aux distances, à 'alimentation, etc.

b) Chaque pays, chaque cité a une situation particulière : des villes sont construites près des frontières, d'autres sur des pentes où souffle un vent plus ou moins continu ; telles villes possèdent des moyens de protection souterrains, telles autres sont entourées de grandes forêts, etc.

$\mathrm{nl}$ faut renseigner le public et surtout les jeunes gens et les personnes qui ne sont pas attachées à l'armée sur ces divers moyens et sur la façon de construire les bâti- 


\section{Sauvegarde des populations et secours aux gazés de guerre.}

ments et de les protéger. Par des tableaux graphiques et par des tracts populaires, on peut faire connaître les bonnes et les mauvaises méthodes de protection passive.

En ce qui concerne plus spécialement l'enseignement de la protection contre les gaz, qui représente une forme nouvelle appliquée de l'enseignement de protection dans les industries, il importe de souligner ici qu'il s'est beaucoup développé en Allemagne depuis plusieurs années. On peut évidemment dans ce domaine se référer aux expériences connues. Dans l'industrie notamment, les moyens efficaces de protection entrent vite en action parce que chacun sait ce qu'il doit faire dès qu'on donne l'alarme.

Du point de vue que nous envisageons, le but qu'on doit s'efforcer d'atteindre est clair : faire jouer le mécanisme de protection dans chaque localité avec la même célérité.

IX.

Le Comité international de la Croix-Rouge ne peut pas suggérer des mesures de protection uniformes étant données les conditions géographiques, économiques et sociologiques dissemblables d'un pays à un autre. D'autre part, la Croix-Rouge ignore les secrets de la défense nationale. Mais, ce qu'il convient à la Croix-Rouge de faire, c'est de synthétiser toutes les connaissances scientifiques et les techniques industrielles connues. On peut en principe charger la Croix-Rouge d'étudier lạ partie mécanique des masques; de favoriser tous les genres d'exercices avec ces masques antigaz; de veiller à la conservation de ces derniers, en se rappelant toutefois que certains caoutchoucs résistent plus ou moins au temps, à la température et à la lumière et qu'il convient de recourir aux meilleurs moyens de conservation. (J'ai des parties de masque qui se sont très bien conservées pendant 15 ans; j'en ai d'autres qui, très fragiles, ont été complètement détruites après deux ou trois ans seulement.) 


\section{Heinrich Zangger.}

$\mathbf{X}$.

L'examen des réponses des Sociétés nationales montre que des Etats, en plus grand nombre qu'on ne le croit généralement, ont promulgué des lois sur la protection de la population civile. Il y en a même qui, pour empêcher la vente de mauvais masques, exigent que tous les masques soient munis de garanties telles que signes de contrôle, estampilles de l'Etat, etc.

Si les Etats mettent à la disposition des organisations de Croix-Rouge des filtres efficaces pour les masques de fuite, e'est probablement tout ce qu'on peut demander.

\section{$\mathrm{XI}$.}

Dans la supposition qu'une ville pourrait être soumise au danger des gaz de guerre durant des heures et même des jours, éventualité effroyable, nous sommes amenés à poser la question suivante : les Sociétés de la Croix-Rouge peuvent-elles déterminer quels sont les moyens qui protégeraient la population civile retenue dans des abris pendant 24 ou 48 heures (production d'oxygène, absorption d'acide carbonique) ?

Les experts réunis à Paris en 1931 pour apprécier le résultat du concours sur la détection de l'ypérite dans l'air ${ }^{1}$ ont toujours insisté sur deux points :

1) Protéger les abris contre les personnes portant des vêtements contaminés, leur faire changer d'habits, désinfecter les vêtements et les souliers avant toute admission dans les abris;

2) Changer l'air, soit par production d'oxygène et absorption, soit par filtration de l'air aspiré.

La situation des personnes entassées dans un abri et menacées d'y manquer d'air demeure une anticipation

1 Voir Revue internationale, avril 1931, pp. 239.243 et mai 1931, p. 359 . 


\section{Sauvegarde des populations et secours aux gazés de guerre.}

terrifiante pour tous ceux qui ont vu et sauvé des personnes enfermées dans des galeries de mines écroulées ou dans des tunnels en construction qui s'étaient effondrés.

Avant la Conférence du désarmement, il était loisible de penser qu'il serait possible de créer un masque muni de garanties internationales. En effet, deux conditions essentielles étaient alors réalisées :

1) Le protocole de Genève du 17 juin 1925 prohibant l'emploi à la guerre des gaz asphyxiants toxiques ou similaires, avec un contrôle efficace, avait été élaboré.

2) Au cas où dans une guerre ce protocole serait violé, on pouvait présumer alors que les gaz de guerre seraient à peu près les mêmes qu'en 1918. En ces années-là, on aurait alors pu créer un masque international. Aujourd'hui, cette idée serait évidemment une absurdité.

La guerre des gaz, telle qu'elle se présenterait en 1935, utiliserait sans doute des moyens d'agression considérés comme des secrets nationaux qui forcent à prévoir dès le temps de paix la protection la plus générale et à ne pas prétendre protéger la population civile uniquement au moyen de masques filtrants individuels très coûteux.

En résumé, quelle entr'aide, quelles mesures de prévoyance le Comité international de la Croix-Rouge peut-il envisager et réaliser de manière continue ?

1) Les Sociétés nationales, si elles tiennent certains travaux pour fort avancés, parfois même comme achevés, tels ceux de la standardisation du matériel sanitaire par exemple, restent dans l'inquiétude quant à la préparation et la systématisation de la défense, du sauvetage et de la thérapeutique dans le domaine des gaz de guerre.

Conformément aux vœux des experts de Bruxelles et de Rome (1928 et 1929), le Comité international a fondé le Centre de documentation, et il est entré de ce fait en 


\section{Heinrich Zangger.}

possession de nombreuses publications, relatives à une guerre des gaz prévisible. Malgré cela, il ne lui a pas été possible de systématiser la protection individuelle puisqu'on reste dans l'ignorance totale des progrès réalisés dans les divers pays. Ce que le Comité international a pu faire, ce fut de réunir à ce sujet des documents et des faits.

Par contre, il lui serait possible de systématiser les données sur le traitement des gazés, en utilisant les expériences faites pendant la guerre, à l'occasion de catastrophes industrielles ou au cours des recherches scientifiques.

Notons que l'organisme humain a, en raison de sa structure et de ses fonctions, des réactions assez restreintes, c'est-à-dire que des substances nocives assez différentes provoquent de sa part des réactions quasi-semblables. Il n'y a pas de thérapeutique spécifique pour les gazés, et l'on ne possède pas pour eux des antidotes spéciaux.

Les poumons absorbent très rapidement toute la masse des gaz toxiques; ceux-ci sont très vite distribués dans tout le corps, où leur action s'exerce en une durée assez égale. Cela contraste avec les infections et les intoxications par la bouche et par l'estomac, dont les effets se produisent par phases successives. Les toxiques de guerre se distribuent et agissent avec une telle rapidité qu'il est impossible de s'en saisir pour les neutraliser à l'aide de substances chimiques spécifiques, contrairement à ce qui se passe pour les toxiques métalliques et métalloïdes.

D'autre part, au moment où les substances toxiques entrent en activité, l'on ne connaît pas leur nature chimique, puisqu'à coup sûr, celui qui recourra à ces moyens d'attaque choisira des substances combinées ou des toxiques nouveaux, ce qui contribuera encore à empêcher l'application d'une thérapeutique chimiquement spécifique. 


\section{Sauvegarde des populations \\ et secours aux gazés de guerre.}

De plus, e'est aussi dans le milieu toxique qu'il faudra donner des soins.

Ces considérations ont conduit le Centre de documentation à rassembler des documents sur la manière de transporter rationnellement les gazés, les dangers à courir durant ce transport étant à peu près les mêmes pour eux que pour les malades atteints de grippe grave.

Les méthodes de premiers secours peuvent être fixées définitivement afin de renseigner le personnel de CroixRouge et les médecins sur les soins qu'il convient de donner dans telle ou telle phase de l'intoxication.

Cette intoxication se produit en peu de temps; les réactions aux toxiques de guerre ayant pour effet un état pathologique dangereux (espèce de pathergie), les gaz produisant surtout l'oedème des poumons, les affaiblissements du cœur, l'augmentation de la viscosité du sang, (thrombose, nécrose).

Les différentes réactions d'intoxication forment une chaîne aux anneaux fortement liés. Dans chaque phase est virtuellement contenue la phase ultérieure ; aussi une thérapeutique bien definie s'impose-t-elle avant que n'apparaissent les symptômes graves : thérapeutique présymptomatique, espèce de thérapeutique préventive.

Dans ce domaine une thérapeutique s'est constituée pour toute une grande classe de gaz de guerre; elle est mieux caractérisée que pour toute autre intoxication (toxiques des poumons).

La thérapeutique doit être préventive, parce que les réactions qui mettent la vie en danger se manifestent presque toujours seulement après une action latente, de durée variable, et parce que les phénomènes physicochimiques sont si bien localisés et définis qu'une médication recourant aux mêmes moyens s'impose.

Ces possibilités sont limitées à un intervalle de temps déterminé, à la phase définie de l'évolution du mal, où l'organisme reste sensible à ces moyens thérapeutiques, 


\section{Heinrich Zangger.}

c'est-à-dire avant que ne se développent des symptômes dangereux; la plupart des intoxiqués sont sauvés, et définitivement, si on leur applique une médication préventive efficace; celle-ci peut aller jusqu'à supprimer les symptômes en cours d'évolution. La vie des intoxiqués est moins en danger que celle des grands blessés dont les plaies sont infectées. La thérapeutique préventive et présymptomatique est applicable, parce que les symptômes sont en état de potentialité, mais ne menacent pas encore effectivement la vie.

Un exemple. Pour les toxiques qui irritent les poumons, la thérapeutique se présente de la manière suivante; il faut :

1) Supprimer les efforts du malade. Tout effort, en effet, exige un accroissement de l'activité des poumons, un massage de ceux-ci, qui est toujours néfaste à la suite de l'intoxication, même avant la réalisation des symptômes dangereux.

2) Protéger la surface respiratoire des poumons contre l'irritation et la dessication en leur procurant de l'air pur anesthésié avec des vapeurs légèrement alcalines, par exemple de la menthe.

3) Prévenir l'oedème des poumons et la perte d'eau du sang. On peut avec les forces osmotiques retenir l'eau dans la circulation en injectant plusieurs fois, par exemple, des solutions de glucose de 30 à $50 \%$ (avec un $\mathrm{cm}^{3} 1 \%$ d'adrénaline et des préparations de strophantine, de digitale). Combiner ces injections avec des saignées si l'oedème des poumons commence à se constituer. Cette thérapeutique a des résultats presque toujours remarquables, même dans les cas d'oedème des poumons, de cyanose, de sang déjà très épais ne coulant plus spontanément à la saignée avant l'injection de glucose, même quand le cour est déjà très affaibli. En voici la raison :

Les intoxiqués ont été des gens jeunes et forts. Les résultats sont, à vrai dire, beaucoup moins favorables pour des personnes plus âgées. D'autres médications, comme les préparations organiques de chaux, par exemple, doivent être appliquées au début, ou quand l'oedème des poumons est en grande partie résorbé.

En résumé : une thérapeutique préventive et présymptomatique de ce genre se combinera très probablement avec la respiration d'un air pas trop froid, mêlé de vapeurs d'eau, l'absorption de tisanes chaudes et le repos complet. Pour les personnes intoxiquées par les gaz de guerre, la thérapeutique est simple et définie (des toxiques 


\section{Sauvegarde des populations et secours aux gazés de, guerre.}

comme l'ypérite et d'autres substances produisent surtout l'irritation, l'inflammation et la nécrose, ot ils sont à redouter en raison de l'infection qu'ils produisent, de la gránde durée de la maladie et des séquelles.)

En conséquence, le Comité international peut réunir, de manière continue, les expériences thérapeutiques; il peut orienter les médecins des Sosiétés nationales de la Croix-Rouge sur les expériences cliniques, sur les expériences faites dans l'industrie non soumises au secret et entrées d'emblée dans le domaine public ; il peut systématiser toutes ces expériences et les communiquer par le moyen du Centre de documentation qui fera ainsi connaître continuellement les modalités d'une action efficace. 